\title{
Mechanism of stress relaxation in Ge nanocrystals embedded in $\mathrm{SiO}_{2}$
}

I.D. Sharp ${ }^{\mathrm{a}, \mathrm{b})}$ D.O. Yi, ${ }^{\mathrm{a}, \mathrm{c})}$ Q. Xu, ${ }^{\mathrm{a}, \mathrm{b})}$ C.Y. Liao, ${ }^{\mathrm{a}, \mathrm{b})}$ J.W. Beeman ${ }^{\text {a) }}$ Z. Liliental-Weber, ${ }^{\mathrm{a})}$ K.M. Yu, ${ }^{\text {a) }}$ D.N. Zakharov, ${ }^{\text {a) }}$ J.W. Ager III, ${ }^{\text {a) }}$ D.C. Chrzan, ${ }^{\mathrm{a}, \mathrm{b}}$ ) and E.E. Haller, ${ }^{\mathrm{a}, \mathrm{b}}$

a) Materials Sciences Division, Lawrence Berkeley National Laboratory, Berkeley, CA 94720

b) Department of Materials Science and Engineering, University of California, Berkeley, CA 94720

${ }^{c}$ Applied Science and Technology, University of California, Berkeley, CA 94720

\begin{abstract}
Ion-beam-synthesized ${ }^{74} \mathrm{Ge}$ nanocrystals embedded in an amorphous silica matrix exhibit large compressive stresses in the as-grown state. The compressive stress is determined quantitatively by evaluating the Raman line shift referenced to the line position of freestanding nanocrystals. Post-growth thermal treatments lead to stress reduction. The stress relief process is shown to be governed by the diffusive flux of matrix atoms away from the local nanocrystal growth region. A theoretical model that quantitatively describes this process is presented.
\end{abstract}

PACS numbers: 81.07.-b; 62.25. $+g ; 65.80 .+n ; 6322 .+m$ 
Stresses in heteroepitaxial thin films and two-dimensional (2-D) islands have been extensively studied and remain an active area of research. Fundamental understanding of these stresses has been used for precise bandgap engineering, ${ }^{1}$ device performance enhancement, ${ }^{2}$ size distribution control, $^{3}$ and self-organization of island arrays. ${ }^{4}$ Similarly, significant stresses have been observed after three-dimensional (3-D) growth of embedded nanocrystals. ${ }^{5,6}$ However, stress generation and relief mechanisms in these 3-D systems are not as well understood. Further understanding of the role of growth stresses in the physical synthesis of 3-D nanocrystal systems is critical to realizing their full potential in future technological applications. In this letter, we report the results of experiments to relieve stress in Ge nanocrystals embedded in silica and present a theoretical model to describe the evolution of stress relaxation during post-growth thermal annealing. We show that stress relaxation is governed by a diffusive process and may therefore be accurately predicted and controlled.

${ }^{74} \mathrm{Ge}$ nanocrystals were formed in a $500 \mathrm{~nm}$ thick thermally grown $\mathrm{SiO}_{2}$ thin film on a $\mathrm{Si}$ wafer substrate. Multi-energy implantation, which provides a relatively constant Ge concentration distribution within the nanocrystal growth region, was performed with ion energies and doses of $50 \mathrm{keV}$ at $1 \times 10^{16} \mathrm{~cm}^{-2}, 80 \mathrm{keV}$ at $1.2 \times 10^{16} \mathrm{~cm}^{-2}$, and $120 \mathrm{keV}$ at $2 \times 10^{16} \mathrm{~cm}^{-2}$. Nanocrystals were grown by annealing in an Ar atmosphere at $900{ }^{\circ} \mathrm{C}$ for 1 hour followed by rapid quenching from the annealing temperature. A cross-sectional transmission electron micrograph of typical as-grown Ge nanocrystals is presented in Fig. 1. As-grown nanocrystals have a mean diameter of $5.1 \mathrm{~nm}$ and a $3.9 \mathrm{~nm}$ size distribution FWHM.

A Raman spectrum from as-grown ${ }^{74} \mathrm{Ge}$ nanocrystals is shown in Fig. 2(a). Asymmetric broadening of the Raman line arising from phonon confinement ${ }^{7}$ is observed. However, instead of the red shift predicted by the phonon confinement model, the Raman line is blue-shifted 
relative to that of the isotopically enriched ${ }^{74} \mathrm{Ge}$ single crystal shown in Fig. 2(c). The blue shift is attributed definitively to external compressive stress by comparing the spectrum of as-grown ${ }^{74} \mathrm{Ge}$ nanocrystals to the spectrum of comparable free-standing Ge nanocrystals obtained by selective etching of the oxide in a 1:1 49\% HF: $\mathrm{H}_{2} \mathrm{O}$ solution. ${ }^{8,9}$ Upon removal of the matrix, compressive stress is relaxed and the nanocrystal Raman line appears at a lower frequency than the single crystal, consistent with phonon confinement. We note that the use of isotopically pure nanocrystals and bulk crystal standards increases the precision of these stress measurements. ${ }^{6}$ No Raman spectral features in the vicinity of $400 \mathrm{~cm}^{-1}$, which would indicate $\mathrm{Si}_{\mathrm{x}} \mathrm{Ge}_{1-\mathrm{x}}$ alloying, are observed for any samples.

To characterize the relaxation process, post-growth annealing was performed for times ranging from 30 minutes to 48 hours at $600{ }^{\circ} \mathrm{C}, 700{ }^{\circ} \mathrm{C}$ and $800{ }^{\circ} \mathrm{C}$. We will present elsewhere the results of in situ electron diffraction and Raman spectroscopy experiments which show conclusively that as-grown Ge nanocrystals embedded in the silica matrix remain solid throughout the post-annealing process (the annealing temperatures are all below the bulk melting point of Ge, $937^{\circ} \mathrm{C}$ ). However, these experiments do not determine whether Ge is solid or liquid during the $900{ }^{\circ} \mathrm{C}$ growth process itself.

Post-growth annealing results in smaller Raman shifts (Fig. 2(b)) that are indicative of reduced compressive stresses and that approach a constant value at long times. Furthermore, the stress reduction rate increases with temperature, suggesting that the stress relief mechanism is a thermally activated process. The viscosity of fused silica, which is orders of magnitude larger that $10^{14} \mathrm{Pas}$ to temperatures exceeding $1000{ }^{\circ} \mathrm{C},{ }^{10}$ is too high to be involved in a mechanism leading to stress relief. 
Raman spectroscopy is used to make accurate and quantitative measurements of the stress states of embedded nanocrystals because the small total volume of nanocrystalline Ge precludes direct measurement of the lattice spacing via x-ray diffraction. The magnitude of the observed compressive stress is determined relative to that of the free-standing crystals using the following equation for the hydrostatic pressure, $P:^{11}$

$$
P=\frac{\omega_{\text {embedded }}-\omega_{\text {etched }}}{3 \gamma \omega_{o}\left(S_{11}+2 S_{12}\right)}
$$

where $\omega_{\text {embedded }}, \omega_{\text {etched }}$, and $\omega_{o}$ are the Raman shifts of stressed nanocrystals embedded in the $\mathrm{SiO}_{2}$ matrix, relaxed nanocrystals after selective removal of the oxide matrix, and the isotopically enriched ${ }^{74} \mathrm{Ge}$ reference sample, respectively. $S_{i j}$ is the $\mathrm{ij}^{\text {th }}$ element of the elastic compliance tensor and $\gamma$ is the mode-Grüneisen parameter. ${ }^{11}$

After the most rigorous post-growth annealing treatment at $800{ }^{\circ} \mathrm{C}$ for 48 hours, the nanocrystal size distribution is only slightly changed, as measured using the AFM technique presented in Ref. 8. The mean nanocrystal diameter increases from $5.1 \mathrm{~nm}$ to $5.5 \mathrm{~nm}$ and the FWHM increases from $3.9 \mathrm{~nm}$ to $4.4 \mathrm{~nm}$. This is the upper limit to the change of the size distribution with post-annealing and could result in an underestimate of the magnitude of pressure relaxation up to $0.14 \mathrm{GPa}$ (within the error bar given in Fig. 3), as calculated by the phonon confinement model. ${ }^{7}$ For samples annealed for shorter times or at lower temperatures, coarsening-induced error is considerably lower and below the precision of stress measurement.

This stress measurement method assumes that the surface tension for the air/Ge and $\mathrm{SiO}_{2} / \mathrm{Ge}$ interfaces are similar, an assumption supported by the fact that no significant difference is observed between the Raman line positions of free-standing nanocrystals and stress-relieved 
embedded nanocrystals produced by 48 hours of post-annealing at $800{ }^{\circ} \mathrm{C}$. Therefore, differences in pressure between the embedded and free-standing nanocrystals arising from the Gibbs-Thomson effect are not resolvable beyond the error of the measurement technique and may be neglected.

The origin of compressive stresses observed here and in other reports ${ }^{5,6}$ for Ge nanocrystals embedded in $\mathrm{SiO}_{2}$ has not been conclusively established. The difference in thermal expansion coefficients of Ge and silica is of the wrong sign to generate the stresses observed. ${ }^{6}$ It has been proposed that $\mathrm{Ge}$ nucleates and grows in the liquid phase, creating a void within the matrix equivalent to the Ge droplet size. ${ }^{6}$ Due to the $5 \%$ volume expansion of Ge upon solidification, the matrix void exerts a compressive stress to accommodate the solid nanocrystal after cooling from the growth temperature. Though this explanation is plausible, further experiments will be required to determine whether Ge is liquid during the growth process.

We have developed a quantitative model describing the mechanism of thermally induced stress relaxation that is independent of the stress generation mechanism. An individual nanocrystal is modeled as an elastically isotropic spherical precipitate of radius $R_{G e}$ confined within a spherical matrix cavity of radius $R_{\mathrm{SiO}_{2}}$ in elastically isotropic silica. The nanocrystal surface is assumed to be in direct contact with the silica at all times. Given these assumptions, the absolute pressure of the nanocrystals can be calculated in terms of the elastic properties of the Ge and the silica, and Ge/silica surface tension:

$$
P_{G e}=\frac{12 B_{G e} \mu_{S_{i O_{2}}}\left[R_{G e}-R_{S_{i O}}\right]+3 B_{G e} 2 \gamma_{G e-S i O_{2}} R_{S i O_{2}} / R_{G e}}{4 \mu_{S i O_{2}} R_{G e}+3 B_{G e} R_{S i O_{2}}}
$$


where $\mu_{\mathrm{SiO}_{2}}$ and $B_{\mathrm{Ge}}$ are the shear modulus of silica and the bulk modulus of Ge, respectively, and $\gamma_{\mathrm{Ge}^{-S i O_{2}}}$ is the solid Ge/silica interface energy. The time evolution of the nanocrystal pressure is governed by the time dependence of the silica cavity radius. Both the silica cavity radius and the nanocrystal radius are dependent on the temperature.

The pressure within the nanocrystals exceeding the pressure due to the surface tension is defined as the initial pressure, $P_{\text {init }}$. The initial pressure creates a thermodynamic driving force for the radius of the cavity in the silica to increase. ${ }^{12}$ We model this increase in cavity radius as resulting from the diffusion of matrix atoms away from the $\mathrm{Ge} /$ silica interface and into the interior of the $\mathrm{SiO}_{2}$. Though all the atomistic details of this diffusion process are not available, we assume that the $\mathrm{O}$ diffuses rapidly, and the stress relaxation is mediated by the slower diffusion of either $\mathrm{Si}$ atoms or $\mathrm{SiO}$ molecules, referred to as interstitial species, through the silica. In the limit for which the growth rate of the cavity is slow in comparison to the rate at which the interstitial concentration profile changes, the equation describing the diffusive process that relieves the stress on the nanocrystal is given by:

$$
\frac{d R_{\mathrm{SiO}_{2}}(t, T)}{d t}=\frac{D_{M}^{0} \exp \left(-\frac{E_{M}}{k_{B} T}\right)}{R_{\mathrm{SiO}_{2}}(t, T)}\left\{\exp \left[\left(P_{G e}-\frac{2 \gamma_{G e-S i O_{2}}}{R_{G e}(T)}\right) \frac{\Omega_{S_{i O_{2}}}}{k_{B} T}\right]-1\right\}
$$

Here, $R_{S_{i O_{2}}}(t, T)$ is the cavity radius at time $t$ and temperature $T, P_{G e}$ is the absolute nanocrystal pressure at time $t$ and temperature $T$ computed from Eq. (2) using the appropriate parameters, and $\Omega_{\mathrm{SiO}_{2}}$ is the atomic volume of a silica molecule, $45.3 \AA^{3}$. The diffusion coefficient that governs cavity expansion is given by $D_{M}^{0} \exp \left(-E_{M} / k_{B} T\right)$, where $E_{M}$ includes the effective energy barrier to matrix interstitial formation and the migration energy, and $D_{M}^{o}$ is the pre-factor of the effective matrix interstitial diffusivity. The time dependent radius computed 
from Eq. (3) is used to calculate the pressure within the nanocrystals. Finally, $R_{G e}(T)$ is the equilibrium radius of the Ge nanocrystals at temperature $T$, ignoring the effects of surface tension. Since Raman measurements are performed at room temperature, the predictions of Eq. (3) are corrected for the relatively small differential thermal expansion of Ge and silica using bulk values $\alpha_{\mathrm{SiO}_{2}}=5.5 \times 10^{-7} \mathrm{~K}^{-1}$ and $\alpha_{\mathrm{Ge}}=6.5 \times 10^{-6} \mathrm{~K}^{-1}$.

Equation (3) is fit to the experimental data using three parameters: $D_{M}^{o}, P_{i n i t}$, and $E_{M}$. The initial pressure corresponds to the relative pressure calculated in Eq. (1), due to the negligible difference between the $\mathrm{Ge} /$ air and $\mathrm{Ge} / \mathrm{SiO}_{2}$ interface energies. Fig. 3 displays the results of fitting the data using Eq. (3). The fitting parameters are: $P_{i n i t}=1.2 \mathrm{GPa}, E_{M}=2.6 \mathrm{eV}$, $D_{M}^{o}=2.0 \times 10^{-8} \mathrm{~cm}^{2} / \mathrm{s}$. From the plot it is apparent that Eq. (3) describes well the time evolution of the pressure, lending strong support to the present interpretation. Data points for high temperature and long time anneals lie slightly above the theoretical model values and may be the result of some coarsening (Fig. 3), as discussed above.

Self diffusion mechanisms in $\mathrm{SiO}_{2}$ have been studied previously. The fastest diffusing species has been found to be $\mathrm{O} .{ }^{13}$ The slowest diffusing species has been proposed to be either $\mathrm{Si}$ or $\mathrm{SiO}$, depending on the stoichiometry and the presence of nearby $\mathrm{Si} / \mathrm{SiO}_{2}$ interfaces. ${ }^{14}$ If $\mathrm{O}$ is the fastest in our case, Si-rich material would be left behind in the vicinity of the nanocrystals. In Si-rich silica Uematsu ${ }^{14}$ has shown that $\mathrm{SiO}$ is the main diffusing species; this may be the limiting process in pressure relief for our case. The diffusivities obtained in the present work, which vary from $10^{-20} \mathrm{~cm}^{2} / \mathrm{s}$ to $10^{-23} \mathrm{~cm}^{2} / \mathrm{s}$ for our range of temperatures, are considerably larger than those reported in previous studies of diffusion under equilibrium or near-equilibrium conditions. ${ }^{15}$ However, our results are in much better agreement with self-diffusion studies 
performed under non-equilibrium conditions. ${ }^{16}$ This suggests that a significant reduction of the formation energy of the diffusing species may occur in the present case due to implantation damage, the $\mathrm{Si} / \mathrm{SiO}_{2}$ interface, stresses within the matrix, or other non-equilibrium conditions specific to the nanocrystal/matrix system.

In summary, we have demonstrated that stresses generated during the 3-D growth of Ge nanocrystals in a silica matrix may be precisely controlled by means of post-growth thermal annealing. The stress relaxation process may be accurately described by modeling the diffusive flux of matrix atoms away from the nanocrystal growth region. Since semiconductor nanocrystals are expected to interact strongly with light due to quantum confinement effects, ${ }^{17}$ it may be possible to precisely engineer radiative absorption and emission spectra using the stress model and experimental techniques presented here. These compressive stresses may also be exploited to achieve self-organization and additional control over nanocrystal size distributions.

\section{ACKNOWLEDGEMENTS}

The authors acknowledge discussions with W. G. Wolfer. I.D.S. acknowledges support from the Intel Robert N. Noyce fellowship. D.O.Y. acknowledges support from the U.C. Berkeley and Luce Foundation Fellowships. Q.X. acknowledges support through a U.C. Berkeley Fellowship. D.C.C. and E.E.H. acknowledge support from the Miller Institute for Basic Research in Science. This work is supported in part by the Director, Office of Science, Office of Basic Energy Sciences, Division of Materials Science and Engineering, of the U.S. Department of Energy under contract No. DE-AC03-76F00098 and in part by U.S. NSF Grant Nos. DMR-0109844 and EEC-0085569. 


\section{FIGURE CAPTIONS}

Fig. 1: Cross-sectional TEM image of ion beam synthesized Ge nanocrystals embedded in $\mathrm{SiO}_{2}$.

Fig. 2: Raman data obtained using the $488 \mathrm{~nm}$ line of an Ar ion laser operating at $150 \mathrm{~mW}$ in a macroscopic optical setup with $5 \mathrm{~cm}^{-1}$ resolution. a) As-grown ${ }^{74} \mathrm{Ge}$ nanocrystals embedded in amorphous $\mathrm{SiO}_{2}$ for which the peak is shifted to higher frequency, b) liberated ${ }^{74} \mathrm{Ge}$ nanocrystals, and c) symmetric Raman peak of a highly enriched ${ }^{74} \mathrm{Ge}$ single crystal.

Fig. 3: Pressure in Ge nanocrystals embedded in silica, measured relative to free-standing nanocrystal samples, as a function of post-growth thermal annealing conditions. Lines are from the model described by Eq. (3) and are fitted to experimental data with three adjustable parameters. The initial pressure is relieved completely for samples annealed at $800{ }^{\circ} \mathrm{C}$ within a few hours. Lower annealing temperatures relieve the growth pressure at slower rates. Error bars are the standard deviation from repeated Raman line position measurements on each sample. 


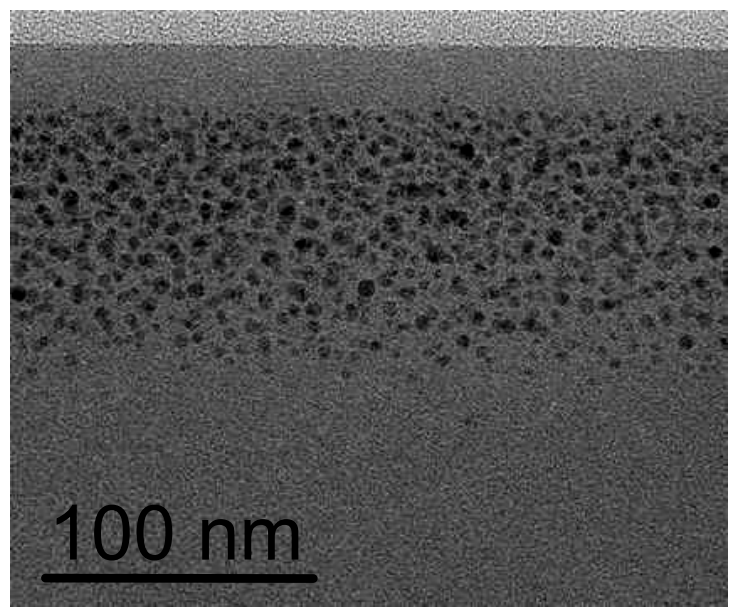

Figure 1 


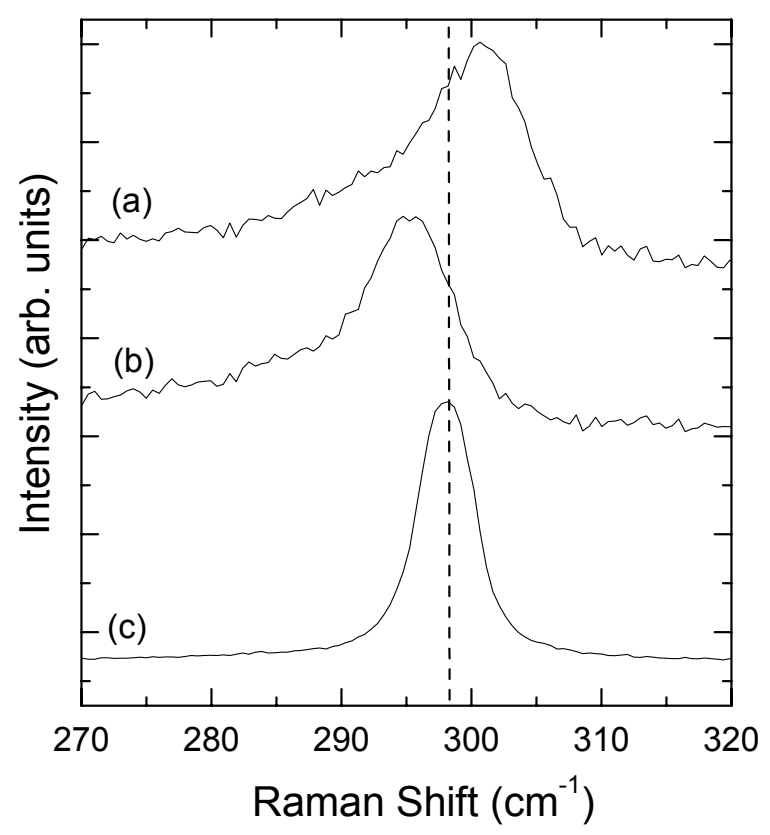

Figure 2 


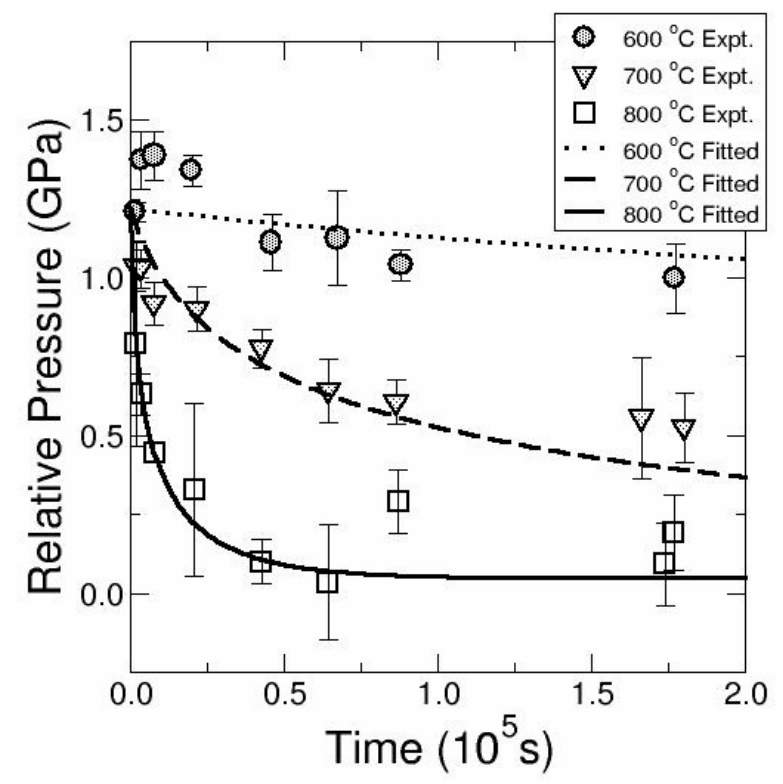

Figure 3 


\section{REFERENCES}

1. M.E. Aumer, S.F. LeBoeuf, S.M. Bedair, M. Smith, J.Y. Lin, and H.X. Jiang, Appl. Phys. Lett. 77, 821 (2000).

2. B.S. Meyerson, Sci. Am. 270, 42 (1994).

3. Zheng Gai, Biao Wu, J. P. Pierce, G. A. Farnan, Dajun Shu, Mu Wang, Zhenyu Zhang, and Jiang Shen, Phys. Rev. Lett. 89, 235502 (2002); Feng Liu, Adam H. Li, and M.G. Lagally, Phys. Rev. Lett. 87, 126103 (2001); C. Priester and M. Lannoo, Phys. Rev. Lett. 75, 93 (1995); M. Meixner, E. Schöll, V. A. Shchukin, and D. Bimberg, Phys. Rev. Lett. 87, $236101(2001)$.

4. V.A. Shchukin, N.N. Ledentsov, P.S. Kop'ev, and D. Bimberg, Phys. Rev. Lett. 75, 2968 (1995); V.A. Shchukin, D. Bimberg, T.P. Munt, and D.E. Jesson, Phys. Rev. Lett. 90, 076102 (2003); J. Tersoff, C. Teichert, and M.G. Lagally, Phys. Rev. Lett. 76, 1675 (1996).

5. Minoru Fujii, Shinji Hayashi, and Keiichi Yamamoto, Jpn. J. Appl. Phys. 30, 687 (1991); A. Cheung, G. de M. Azevedo, C.J. Glover, D.J. Llewellyn, R.G. Elliman, G.J. Foran, and M.C. Ridgeway, Appl. Phys. Lett. 84, 278 (2004); M. Dubiel, H. Hofmeister, E. Schurig, E. Wendler, and W Wesch, Nucl. Instrum. Methods B 166-167, 871 (2000); X.L. Wu, T. Gao, X.M. Bao, F. Yan, S.S. Jiang, and D. Feng, J. Appl. Phys. 82, 2704 (1997); W.K. Choi, V. Ng, S.P. Ng, H.H. Thio, Z.X. Shen, and W.S. Li, J. Appl. Phys. 86, 1398 (1999); U. Serincan, G. Kartopu, A. Guennes, T.G. Finstad, R. Turan, Y. Ekinci, and S.C. Bayliss, Semicond. Sci. Technol. 19, 247 (2004). 
6. A. Wellner, V. Paillard, C. Bonafos, H. Coffin, and A. Claverie, J. Appl. Phys. 94, 5639 (2003).

7. H. Richter, Z.P. Wang, and B. Ley, Solid State Commun. 39, 625 (1981).

8. I.D. Sharp, Q. Xu, C.Y. Liao, D.O. Yi, J.W. Ager III, J.W. Beeman, K.M. Yu, D.N. Zakharov, Z. Liliental-Weber, D.C. Chrzan, and E.E. Haller, Mat. Res. Soc. Symp. Proc. 818, M13.3.1 (2004).

9. We will show elsewhere that removal of the $\mathrm{SiO}_{2}$ matrix does not significantly alter the nanocrystal size distribution.

10. E.B. Shand, in Modern Materials (Academic Press, New York, 1968), Vol. 6, pp. 262.

11. F. Cerdeira, C. J. Buchenauer, Fred H. Pollak, and Manuel Cardona, Phys. Rev. B 5, 580 (1972).

12. G.W. Greenwood, A.J.E. Foreman, and D.E. Rimmer, J. Nucl. Mater. 4, 305 (1959).

13. J.C. Mikkelsen, Appl. Phys. Lett. 45, 1187 (1984).

14. M. Uematsu, H. Kageshima, Y. Takahashi, S. Fukatsu, K.M. Itoh, K. Shiraishi, and U. Gosele, Appl. Phys. Lett. 84, 876 (2004).

15. G. Brebec, R. Seguin, C. Sella C, J. Bevenot, and J.C. Martin, Acta. mater. 28, 327 (1980); Tomonori Takahashi, Shigeto Fukatsu, Kohei M. Itoh, Masashi Uematsu, Akira Fujiwara, Hiroyuki Kageshima, and Yasuo Takahashi, J. Appl. Phys. 93, 3674 (2003).

16. G.K. Celler and L.E. Trimble, Appl. Phys. Lett. 54, 1427 (1989); D. Tsoukalas, C. Tsamis, and J. Stoemenos, Appl. Phys. Lett. 63, 3167 (1993).

17. Toshihide Takagahara and Kyozaburo Takeda, Phys. Rev. B 46, 15578 (1992). 